\title{
THE INTERSECTION TOPOLOGY W.R.T. THE REAL LINE AND THE COUNTABLE ORDINALS
}

\author{
G. M. REED
}

\begin{abstract}
If $\Upsilon_{1}$ and $\Upsilon_{2}$ are topologies defined on the set $X$, then the intersection topology w.r.t. $\Upsilon_{1}$ and $\Upsilon_{2}$ is the topology $\Upsilon$ on $X$ such that $\left\{U_{1} \cap U_{2} \mid U_{1} \in \Upsilon_{1}\right.$ and $\left.U_{2} \in \Upsilon_{2}\right\}$ is a basis for $(X, \Upsilon)$. In this paper, the author considers spaces in the class $\mathscr{C}$, where $(X, \Upsilon) \in \mathscr{C}$ iff $X=\left\{x_{\alpha} \mid \alpha<\omega_{1}\right\} \subseteq \mathbf{R}, \Upsilon_{\mathbf{R}}$ is the inherited real line topology on $X, \Upsilon_{\omega_{1}}$ is the order topology on $X$ of type $\omega_{1}$, and $\Upsilon$ is the intersection topology w.r.t. $\Upsilon_{\mathbf{R}}$ and $\Upsilon_{\omega_{1}}$. This class is shown to be a surprisingly useful tool in the study of abstract spaces. In particular, it is shown that: (1) If $X \in \mathscr{E}$, then $X$ is a completely regular, submetrizable, pseudo-normal, collectionwise Hausdorff, countably metacompact, first countable, locally countable space with a base of countable order that is neither subparacompact, metalindelöf, cometrizable, nor locally compact. (2) (MA $+\neg_{\neg} \mathrm{CH}$ ) If $X \in \mathscr{C}$, then $X$ is perfect. (3) There exists in ZFC an $X \in \mathscr{C}$ such that $X$ is not normal. (4) (CH) There exists $X \in \mathscr{C}$ such that $X$ is collectionwise normal and $\omega_{1}$-compact but not perfect.
\end{abstract}

I. Introduction and main results. If $\Upsilon_{1}$ and $\Upsilon_{2}$ are topologies defined on the set $X$, then $\Upsilon$ is the intersection topology w.r.t. $\Upsilon_{1}$ and $\Upsilon_{2}$ defined on $X$, where $\left\{U_{1} \cap U_{2} \mid U_{1}\right.$ $\in \Upsilon_{1}$ and $\left.U_{2} \in \Upsilon_{2}\right\}$ is a basis for $\Upsilon$. Whereas particular applications of this construction technique have been made previously in the literature (notably by $\mathbf{R}$. Pol to construct a normal, locally metrizable, nonparacompact space [Po], by E. Pol and R. Pol to construct a hereditarily normal, strongly zero-dimensional space with a subspace of positive dimension [PP] and by Hajnal and Juhász to construct (nonregular) $S$ - and $L$-spaces $[\mathbf{H J}]$ ), there has evidently never been a general study of its properties. Over the past few years, the author has intermittently pursued such a study. Preliminary results have been announced concerning $Q$-sets $\left[\mathbf{R}_{1}\right], S$ - and $L$-spaces $\left[\mathbf{R}_{2}\right]$, and normality conditions $\left[\mathbf{R}_{3}\right]$. The author had intended to publish these and related results together in a study of the intersection topology and its applications. However, since such publication continues to recede into the future, and since there has been considerable interest expressed in seeing the results in this paper, the author has decided to present them at this time. These results were first reported at the 1978 Topology Conference at the University of Oklahoma.

The class $\mathscr{C}$. Let $\mathscr{C}$ denote the class of topological spaces such that $(X, \mathscr{C}) \in \mathscr{C}$ iff $X=\left\{x_{\alpha} \mid \alpha<\omega_{1}\right\} \subseteq \mathbf{R}$ and $\Upsilon$ is the intersection topology w.r.t. the inherited real line topology $\Upsilon_{R}$ on $X$ and the order topology $\Upsilon_{\omega_{1}}$ on $X$ of type $\omega_{1}$.

Received by the editors May 16, 1985 and, in revised form, October 1, 1985. Presented to the Society, January 10, 1986 in New Orleans.

1980 Mathematics Subject Classification. Primary 54A10, 54D18; Secondary 54A35, 54D15, 54D20.

Key words and phrases. Intersection topology, collectionwise normal, collectionwise Hausdorff, subparacompact, submetrizable, separable metric spaces. 
TheOrem 1. If $(X, \Upsilon) \in \mathscr{C}$, then $(X, \Upsilon)$ is a completely regular, first countable, locally countable, submetrizable, collectionwise Hausdorff, pseudo-normal, countably metacompact space with a base of countable order and $a G_{\delta}$-diagonal. However, $(X, \Upsilon)$ is neither $\theta$-refinable, subparacompact, metalindelöf, cometrizable, nor locally compact.

THEOREM 2. (MA $+\neg \mathrm{CH})$ If $(X, \Upsilon) \in \mathscr{C},(X, \Upsilon)$ is perfect.

THEOREM 3. If $(X, \Upsilon) \in \mathscr{C}, T F A E$

(1) $(X, \Upsilon)$ is strongly collectionwise Hausdorff.

(2) $(X, \Upsilon)$ is normal.

(3) $(X, \Upsilon)$ is collectionwise normal.

(4) $(X, \Upsilon)$ is countably paracompact.

EXAmple 1. $\exists(X, \Upsilon) \in \mathscr{C}$ which is not normal.

EXAmple 2. (CH) $\exists(X, \Upsilon) \in \mathscr{C}$ which is collectionwise normal and $\omega_{1}$-compact but not perfect.

REMARK. All results (and proofs) about the class $\mathscr{C}$ in this paper remain valid for any separable metric topology intersected with an $\omega_{1}$-type order topology.

II. Applications and comparisons. For topologists studying the structure of abstract spaces, the standards for comparison are the real line and the countable ordinals with the order topology. Modifications of the topology on the real line such as the Michael line and the Sorgenfrey line, and modifications of the topology on the countable ordinals such as the Ostaszewski line and the Kunen line have led to major advancements in our understanding of topological structure. The class $\mathscr{C}$ and analogous intersections between existing spaces, provide a variety of new "lines."

Pol's space. In [Po], R. Pol gave a beautiful example that settled many long outstanding questions in topology. Pol's space was the first to be (1) perfectly normal, locally metrizable, and not metrizable, (2) collectionwise normal with a $G_{\delta}$-diagonal and not paracompact, and (3) a perfect $T_{3}$-space but not subparacompact. The construction technique developed by Pol, although not explicitly utilized as such, was essentially the intersection topology w.r.t. the countable ordinals with the order topology and the Baire space of weight $\omega_{1}$. The results in this paper were motivated by Pol's work.

Note that members of the class $\mathscr{C}$ provide simple (although set-theoretic) analogs to Pol's quite complex space. Under $\mathrm{CH}$, Example 1 is a collectionwise normal with a $G_{\delta}$-diagonal and not paracompact. In addition, it is the first such space simultaneously to have cardinality $\omega_{1}$ and to be $\omega_{1}$-compact. Under $\mathrm{MA}+\neg \mathrm{CH}$, each space in $\mathscr{C}$ is a perfect $T_{3}$-space that is not subparacompact. Question ${ }^{1}$ : The author does not know if there is a perfectly normal space in $\mathscr{C}$. In fact, the author does not know if there is either a normal or a perfect member of $\mathscr{C}$ in ZFC. Using the

${ }^{1}$ This question has now been answered by Ken Kunen. See "Added in revision." 
techniques of $\S I I I$, it can be shown that a space $(X, \Upsilon)$ in $\mathscr{C}$ is perfect iff each $\sigma$-discrete set in $(X, \Upsilon)$ is also the union of countably many closed and discrete subsets.

Collectionwise Hausdorff, nonnormal, first countable $T_{3}$-spaces. The search for a collectionwise Hausdorff, nonnormal first countable $T_{3}$-space (in particular, a Moore space) was apparently begun by R. L. Moore in the 1940's. Although a Moore-space example was announced in 1964 [Wo], the first example that was even first countable and which was available for study was presented in $1975\left(\left[\mathbf{R}_{\mathbf{4}}\right]\right.$, see $\left.\left[\mathbf{R}_{\mathbf{5}}\right]\right)$. Example 1 is another instance of such a space. In addition, it is the first submetrizable example to be locally separable or to have cardinality $\omega_{1}$. In addition, under $\mathrm{MA}+\neg_{\neg} \mathrm{CH}$, it is perfect. Recall that collectionwise Hausdorff, locally separable Moores spaces are metrizable [Wo, AP]. Examples of collectionwise Hausdorff, nonnormal Moore spaces have now been published in $\left[\mathbf{W a}, \mathbf{A P}, \mathbf{R}_{\mathbf{5}}\right]$.

Normal nonmetrizable Moore spaces. In $\left[\mathbf{R}_{\mathbf{6}}\right]$, the author described a technique for associating two Moore spaces $X\left(X_{0}\right)$ and $S\left(X_{0}\right)$ to each first countable $T_{3}$-space $X_{0}$ such that (i) $X\left(X_{0}\right)$, the first stage in the iterated construction of $S\left(X_{0}\right)$, is a closed subset of $S\left(X_{0}\right)$, and (ii) there exists an open, continuous, countable-to-one function from $S\left(X_{0}\right)$ onto $X_{0}$. This technique has proven extremely useful in producing counterexamples in the theory of Moore spaces (eg, $\left.\left[\mathbf{R}_{\mathbf{6}}-\mathbf{R}_{\mathbf{1 0}}\right]\right)$. It is easy to verify that for certain spaces $X_{0}$ (for example, a $Q$-set $=$ subspace of the real line such that each of its subsets is a relative $G_{\delta}$-set), $X\left(X_{0}\right)$ is a normal, nonmetrizable Moore space. However, it has been unresolved until now as to whether the normality of $X\left(X_{0}\right)$ implies the normality of $S\left(X_{0}\right)$. With Example 1, we can now resolve this issue quite easily.

Let $(X, \Upsilon)$ be the nonnormal, first countable $T_{3}$-space of Example 1. From the constructions in $\left[\mathbf{R}_{\mathbf{6}}\right], X(X, \Upsilon), X\left(X, \Upsilon_{\mathbf{R}}\right)$, and $X\left(X, \Upsilon_{\omega_{1}}\right)$ are each topological spaces defined on $H \cup K$, where $H$ is closed and discrete in each topology and $K$ is isolated in each topology. Furthermore, $X(X, \Upsilon)$ is simply the intersection topology w.r.t. $X\left(X, \Upsilon_{\mathbf{R}}\right)$ and $X\left(X, \Upsilon_{\omega_{1}}\right)$. Now, under MA $+\neg \mathrm{CH},\left(X, \Upsilon_{\mathbf{R}}\right)$ is a $Q$-set [T], and hence $X\left(X, \Upsilon_{\mathbf{R}}\right)$ is normal. (Note that basic open sets for points in $H$ in $X\left(X, \Upsilon_{\mathbf{R}}\right)$ can be chosen to be contained in tangent disc neighborhoods.) Clearly, $X(X, \Upsilon)$ must also be normal since any two subsets of $H$ which can be separated in $X\left(X, \Upsilon_{\mathbf{R}}\right)$ can also be separated in $X(X, \Upsilon)$. However, it can easily be established (using Lemma 15 below) that the normality of $S(X, \Upsilon)$ would imply the normality of $(X, \Upsilon)$. Thus $S(X, \Upsilon)$ is not normal.

Finally, it follows by methods introduced below that for each $(X, \Upsilon)$ in $\mathscr{C}$, $X(X, \Upsilon)$ is a submetrizable, noncometrizable Moore space of cardinality $\omega_{1}$. The only other known spaces of this type (due to $\mathrm{H}$. Cook [Co]) were constructed to provide examples of normal Moore spaces whose squares are not normal. Questions. ${ }^{2}$ Under MA $+\neg \mathrm{CH}$, is the square of $X(X, \Upsilon)$ not normal for each $(X, \Upsilon) \in \mathscr{C}$ ? In particular, if $(X, \Upsilon)$ is the space of Example 1, is the square of $X(X, \Upsilon)$ normal and

\footnotetext{
${ }^{2}$ See Added in proof.
} 
can $X(X, \Upsilon)$ be embedded in a separable normal Moore space (see [Pr])? In general, is $S\left(X_{0}\right)$ normal iff the square of $X\left(X_{0}\right)$ is normal for each first countable $T_{3}$-space $X_{0}$ ?

III. Properties shared by all members of $\mathscr{C}$. In the remainder of the paper, we shall assume certain well-known lemmas about the countable ordinals with the order topology. These results will be frequently utilized without explicit mention.

Definition. A subset $H$ of $\omega_{1}$ is stationary provided $H \cap \bar{K} \neq \varnothing$ in the order topology on $\omega_{1}$ for each uncountable subset $K$ of $\omega_{1}$.

Countable Decomposition Lemma [U]. If $H=\bigcup_{i<\omega} H_{i}$ is stationary in $\omega_{1}$, then $\exists i<\omega$ such that $H_{i}$ is also stationary.

Pressing Down Lemma [F]. If $H$ is stationary in $\omega_{1}$ and $f$ is a function from $H$ to $\omega_{1}$ such that $f(\alpha)<\alpha$ in $\omega_{1}$ for each $\alpha \in H$, then $\exists \beta<\omega_{1}$ such that $\{\alpha \in H \mid f(\alpha)$ $=\beta\}$ is stationary in $\omega_{1}$.

Notation. If $(X, \Upsilon) \in \mathscr{C}$, then we assume an $\omega_{1}$-type order on $X$, where $X=\left\{x_{\alpha} \mid \alpha<\omega_{1}\right\}$. By $\left(X, \Upsilon_{\omega_{1}}\right)$, we mean the topology on $X$ induced by this order; by $\left(X, \Upsilon_{\mathbf{R}}\right)$, we mean the inherited real line topology on $X$. We will say that a subset of $X$ is stationary w.r.t. $\left(X, \Upsilon_{\omega_{1}}\right)$ or $\sigma$-discrete w.r.t. $\left(X, \Upsilon_{\omega_{1}}\right)$ provided it has the corresponding property in the order topology. For $x, y \in X$, we will say that $x<y$ in $\omega_{1}$ provided $\beta<\alpha$, where $x=x_{\beta}$ and $y=x_{\alpha}$.

If $(X, \Upsilon) \in \mathscr{C}, \mathscr{B}_{\mathbf{R}}=\left\{B_{i} \mid i<\omega\right\}$ will denote a countable basis for $\left(X, \Upsilon_{\mathbf{R}}\right)$. Furthermore, for each $x=x_{\alpha} \in X$ such that $\alpha$ is a limit ordinal, $\left\{\alpha_{i}\right\}_{i<\omega}$ will denote a fixed sequence converging to $\alpha$ in $\omega_{1}$ such that for each $i<\omega, \alpha_{i}<\alpha_{i+1}<$ $\alpha$. Finally, if $x=x_{\alpha} \in X, \mathscr{G}_{\omega_{1}}(x)$ will denote a local base for $x$ in $\left(X, \Upsilon_{\omega_{1}}\right)$, where $\mathscr{G}_{\omega_{1}}(x)=\{\{x\}\}$ if $\alpha$ is a nonlimit ordinal, and $\mathscr{G}_{\omega_{1}}(x)=\left\{G_{i}(x) \mid i<\omega\right\}$, where $G_{i}(x)=\left\{x_{\beta} \mid \alpha_{i}<\beta \leqslant \alpha\right\}$, if $\alpha$ is a limit ordinal.

A point set $K$ in the space $X$ is said to be discrete provided no point of $K$ is a limit point of $K$. A collection $\mathscr{H}$ of point sets is said to be discrete provided for each $x \in X, \exists$ an open set $\nu(x)$ containing $x$ such that $|\{H \in \mathscr{H} \mid \nu(x) \cap H\} \neq \varnothing| \leqslant 1$.

LEMMA 1. If $\left(X, \Upsilon_{1}\right)$ and $\left(X, \Upsilon_{2}\right)$ are both metrizable, then so is $(X, \Upsilon)$ where $\Upsilon$ is the intersection topology w.r.t. $\Upsilon_{1}$ and $\Upsilon_{2}$.

Proof. Let $\mathscr{B}=\left\{\mathscr{B}_{i} \mid i<\omega\right\}$ and $\mathscr{G}=\left\{\mathscr{G}_{j} \mid j<\omega\right\}$ denote $\sigma$-discrete bases for $\left(X, \Upsilon_{1}\right)$ and $\left(X, \Upsilon_{2}\right)$, respectively. It follows immediately that

$$
V=\bigcup_{i<\omega} \bigcup_{j<\omega}\left\{B \cap G \mid B \in \mathscr{B}_{i} \text { and } G \in \mathscr{G}_{j}\right\}
$$

is a $\sigma$-discrete base for $(X, \Upsilon)$.

LeMma 2. If $(X, \Upsilon) \in \mathscr{C}$, then $(X, \Upsilon)$ is a completely regular, first countable, locally countable, submetrizable (hence, with a $G_{\delta}$-diagonal) space.

Proof. Straightforward. 
Lemma 3. If $(X, \Upsilon) \in \mathscr{C}$ and $K \subseteq X, T F A E$

(1) $K$ is $\sigma$-discrete in $(X, \Upsilon)$.

(2) $K$ is o-discrete in $\left(X, \Upsilon_{\omega_{1}}\right)$.

(3) $K$ is not stationary in $\left(X, \Upsilon_{\omega_{1}}\right)$.

(4) $K$ is a metrizable subspace of $\left(X, \Upsilon_{\omega_{1}}\right)$.

(5) $K$ is a metrizable subspace of $(X, \Upsilon)$.

(6) $\exists$ an open, $\sigma$-discrete, metrizable subspace of $\left(X, \Upsilon_{\omega_{1}}\right)$ containing $K$.

(7) $\exists$ an open, $\sigma$-discrete, metrizable subspace $(X, \Upsilon)$ containing $K$.

Proof. (1) $\rightarrow$ (3). Suppose $K=\bigcup_{i<\omega} K_{i}$, where for each $i<\omega, K_{i}$ is discrete in $\left(X, \Upsilon_{\omega_{1}}\right)$ and $K$ is stationary in $\left(X, \Upsilon_{\omega_{1}}\right)$. Then, $\exists m$ such that $K_{m}$ is stationary in $\left(X, \Upsilon_{\omega_{1}}\right)$. For each $x \in K_{m}, \exists U(x)=G(x) \cap B_{i x}$ such that $G(x) \in \mathscr{G}_{\omega_{1}}(x), x \in B_{i_{x}}$ $\in \mathscr{B}_{\mathbf{R}}$, and $U(x) \cap\left(K_{m} \backslash\{x\}\right)=\varnothing$. Thus, $\exists n$ such that $K_{m, n}=\left\{x \in k_{m} \mid i_{i_{x}}=\right.$ $n\} \subseteq B_{n}$ is stationary in $\left(X, \Upsilon_{\omega_{1}}\right)$. Hence, $\exists y \in K_{m, n}$ such that $y$ is a limit point of $K_{m, n}$ in $\left(X, \Upsilon_{\omega_{1}}\right)$. However, $\left(U(y) \cap B_{n}\right) \cap\left(K_{m} \backslash\{y\}\right) \neq \varnothing$, which is a contradiction.

(2) $\leftrightarrow(3) \leftrightarrow(4)$. [vDL].

$(2) \rightarrow(6)$. Let $K=\bigcup_{i<\omega} K_{i}$, where each $K_{i}$ is discrete in $\left(X, \Upsilon_{\omega_{1}}\right)$. Since $\left(X, \Upsilon_{\omega_{1}}\right)$ is hereditarily collectionwise Hausdorff and locally countable, $\exists$ an open, $\sigma$-discrete subset $U$ of $\left(X, \Upsilon_{\omega_{1}}\right)$ containing $K$. Since $(2) \leftrightarrow(4), U$ is an open metrizable subspace of $\left(X, \Upsilon_{\omega_{1}}\right)$.

$(6) \rightarrow$ (7). Follows from Lemma 1.

(7) $\rightarrow(5)$. Obvious.

$(5) \rightarrow(1)$. Suppose $Y$ is a metrizable subspace of $(X, \Upsilon)$. Then $Y$ is metalindelöf. Each first countable, locally countable, metalindelöf space is the disjoint union of a collection of open and closed countable subsets. Hence, $Y$ is $\sigma$-discrete.

Lemma 4. Suppose $(X, \Upsilon) \in \mathscr{C}$ and $B$ is an open set in $\left(X, \Upsilon_{\mathbf{R}}\right)$ containing the stationary set $H$ w.r.t. $\left(X, \Upsilon_{\omega_{1}}\right)$, and for each $x \in X, G(x) \in \mathscr{G}_{\omega_{1}}(x)$. If $U$ is the open set in $(X, \Upsilon)$ such that $U=\bigcup\{G(x) \cap B \mid x \in H\}$, then $|B \backslash U|<\omega_{1}$.

Proof. Suppose $U$ and $B$ are as described and $|B \backslash U|=\omega_{1}$. Then $B \backslash U$ has a limit point $x \in H$ w.r.t. $\left(X, \Upsilon_{\omega_{1}}\right)$. Hence, $(B \backslash U) \cap(G(x) \cap B) \neq \varnothing$, which is a contradiction.

LeMmA 5. If $(X, \Upsilon) \in \mathscr{C}$, then $(X, \Upsilon)$ is pseudo-normal and collectionwise Hausdorff.

Proof. (1) A space is pseudo-normal provided each two disjoint closed subsets, one of which is countable, can be separated by open sets. Let $H, K$ be two such sets in $(X, \Upsilon)$, where $K$ is countable. Clearly, $(X, \Upsilon)=U \cup V$, where $U \cap V=\varnothing, U$ and $V$ are both open, $U$ is countable, and $K \subseteq U$. Since each countable, regular first countable space is metrizable, $K$ and $U \cap H$ can be separated by open subsets of $U$. Hence, $K$ and $H$ can be separated by open sets in $(X, \Upsilon)$.

(2) A space is collectionwise Hausdorff provided each closed and discrete subset $H$ can be screened by open sets, i.e. $\exists$ a pairwise disjoint collection $\mathscr{V}$ of open sets covering $H$ such that for each $V \in \mathscr{V},|V \cap H|=1$. Let $H$ be a closed and discrete 
subset of $(X, \Upsilon)$. By Lemma 3, $\exists$ an open metrizable subset $U$ of $(X, \Upsilon)$ containing $H$. Hence, since each metrizable space is collectionwise Hausdorff, $H$ can be screened in $U$, and thus in $(X, \Upsilon)$.

LeMma 6. If $(X, \Upsilon) \in \mathscr{C}$, then $(X, \Upsilon)$ is countably metacompact.

Proof. A space is countably metacompact provided each countable open covering has a point-finite open refinement that covers the space. Let $\mathscr{D}=\left\{D_{i} \mid i<\omega\right\}$ be an open covering of $(X, \Upsilon)$. For each $x \in X, \exists G(x) \in \mathscr{G}_{\omega_{1}}(x)$ and $B(x) \in \mathscr{B}_{\mathbf{R}}$ such that $U(x)=G(x) \cap B(x)$ is contained in an element of $\mathscr{D}$. For each $i<\omega$ and $j<\omega$, let $X_{i j}=\left\{x \in X \mid B(x)=B_{i}\right.$ and $\left.U(x) \subseteq D_{j}\right\}$, and let

$$
U_{i j}=\bigcup\left\{U(x) \mid x \in X_{i j}\right\} \text {. }
$$

For each $B=B_{i} \in \mathscr{B}_{\mathbf{R}}$ such that $X_{i j}$ is stationary for some $j<\omega$, let $X(B)=X_{i j}$, and let $U(B)=U_{i j}$. Note by Lemma $4,|B \backslash U(B)|<\omega_{1}$. Let $\mathscr{W}=$ the set of all such $B$. Consider $\bigcup \mathscr{W}$ as a subspace of $\left(X, \Upsilon_{\mathbf{R}}\right)$ and let $\mathscr{F}$ be a countable point-finite open refinement of $\mathscr{W}$ covering $\bigcup \mathscr{W}$. Now for each $F \in \mathscr{F}$, let $V(F)=$ $F \cap U(B)$ for a fixed $B \in \mathscr{W}$ such that $F \subseteq B$. Clearly, $\mathscr{V}=\{V(F) \mid F \in \mathscr{F}\}$ is a point-finite open refinement of $\mathscr{D}$ in $(X, \Upsilon)$. Furthermore, by construction, $K=$ $X \backslash \cup \mathscr{V}$ is $\sigma$-discrete. Thus, by Lemma 3, $\exists$ an open $\sigma$-discrete, metrizable subset $Y$ of $(X, \Upsilon)$ containing $K$. Hence, let $\mathscr{V}^{\prime}$ denote a point-finite collection of open sets in $(X, \Upsilon)$ which refines $\{D \cap Y \mid D \in \mathscr{D}\}$. It follows that $\mathscr{V} \cup \mathscr{V}^{\prime}$ is the desired point-finite collection for $(X, \Upsilon)$.

LEMma 7. If $(X, \Upsilon) \in \mathscr{C}$, then $(X, \Upsilon)$ has a base of countable order.

Proof. From Lemma $2,(X, \Upsilon)$ is locally metrizable. Each locally metrizable space has a base of countable order [WW].

LEMma 8. If $(X, \Upsilon) \in \mathscr{C}$, then $(X, \Upsilon)$ is not $\theta$-refinable.

Proof. Since each $\theta$-refinable $T_{3}$-space with a base of countable order is a Moore space [WW] and each Moore space is subparacompact [B], it suffices by Lemma 7 to show that $(X, \Upsilon)$ is not subparacompact. A space is subparacompact provided each open covering has a $\sigma$-discrete refinement of closed sets covering the space. Hence, since $(X, \Upsilon)$ is locally countable, it would be $\sigma$-discrete were it subparacompact. This would contradict Lemma 3.

Lemma 9. If $(X, \Upsilon) \in \mathscr{C}$ and $K \subseteq X$ such that each infinite subset of $K$ has a limit point in $(X, \Upsilon)$, then $\bar{K}$ is a compact, metrizable subspace of $(X, \Upsilon)$ and $|\bar{K}|<\omega_{1}$.

Proof. (1) $\bar{K}$ is a compact and metrizable subspace of $(X, \Upsilon)$. Since each countably compact $T_{3}$-space with a $G_{\delta}$-diagonal is metrizable [Ch], we need only to show that $\bar{K}$ is countably compact. Hence, suppose $J$ is an infinite subset of $\bar{K} / K$ such that $J$ has no limit point in $(X, \Upsilon)$. Let $x \in X$ such that $x$ is a limit point of $J$ in $\left(X, \Upsilon_{\omega_{1}}\right)$ and let $J^{\prime}=\left\{x_{i} \mid i<\omega\right\} \subseteq J$ such that $\left\{x_{i}\right\}_{i<\omega}$ converges to $x$ in $\left(X, \Upsilon_{\omega_{1}}\right)$. Now, for each $0<i<\omega$, let $y_{i} \in K$ such that $x_{i-1}<y_{i}<x_{i}$ in $\omega_{1}$ and $d\left(x_{i}, y_{i}\right)<1 / i$ in $(X, \Upsilon)$. Clearly, $\left\{y_{i}\right\}_{i<\omega}$ also converges to $x$ in $\left(X, \Upsilon_{\omega_{1}}\right)$. Hence 
$\left\{y_{i}\right\}_{i<\omega}$ must converge to $x$ in $(X, \Upsilon)$ since there is no other possibility. However, it follows immediately that $x$ is a limit point of $J^{\prime}$ in $(X, \Upsilon)$, and we have our contradiction.

(2) $|\bar{K}|<\omega_{1}$. By Lemma 3, $\bar{K}$ is $\sigma$-discrete since it is metrizable. Each $\sigma$-discrete, compact, metrizable space is obviously countable.

Lemma 10. If $(X, \Upsilon) \in \mathscr{C}$, then $(X, \Upsilon)$ is not locally compact.

Proof. Suppose $(X, \Upsilon) \in \mathscr{C}$ and $(X, \Upsilon)$ is locally compact. For each $x \in X$, let $U(x)=G(x) \cap B(x)$, where $G(x) \in \mathscr{G}_{\omega_{1}}(x), x \in B(x) \in \mathscr{B}_{\mathbf{R}}$, and $\overline{U(x)}$ is compact in $(X, \Upsilon)$. By the Pressing Down Lemma, $\exists \gamma<\omega_{1}$ and a stationary subset $Y$ of $X$ in $\left(X, \Upsilon_{\omega_{1}}\right)$ such that for all $x=x_{\alpha} \in Y, G(x)=\left\{x_{\beta} \mid \gamma<\beta \leqslant \alpha\right\}$. Furthermore, $\exists B \in \mathscr{B}_{\mathrm{R}}$ such that $B=B(x)$ for each $x$ in a stationary subset $Y^{\prime}$ of $Y$. Consider $U=\bigcup\left\{U(x) \mid x \in Y^{\prime}\right\}$. Suppose $J$ is an infinite subset of $U$, then $\exists x \in Y^{\prime}$ such that $J \subseteq U(x)$. Hence $J$ has a limit point in $(X, \Upsilon)$. Thus, by Lemma $9,|U|<\omega_{1}$ and we have a contradiction to the fact that $Y^{\prime}$ is stationary in $\left(X, \Upsilon_{\omega_{1}}\right)$.

Lemma 11. If $(X, \Upsilon) \in \mathscr{C}$, then $(X, \Upsilon)$ is not metalindelöf.

Proof. See the proof of $(5) \rightarrow(1)$ in Lemma 3.

LeMma 12. If $(X, \Upsilon) \in \mathscr{C}$, then $(X, \Upsilon)$ is not cometrizable.

Proof. A function $f$ from the space $X$ to the space $S$ is said to be regular provided that for each $x \in X$ and open set $U$ containing $x$, there exists an open set $V$ in $X$ such that $x \in V$ and if $y \in \overline{f(V)}$ in $S$, then $f^{-1}(y) \in U$. A $T_{2}$-space $X$ is cometrizable iff there exists a regular, one-to-one, continuous function from $X$ onto a separable metric space.

Let $(X, \Upsilon) \in \mathscr{C}$ and suppose $f$ is a regular, one-to-one, continuous function from $(X, \Upsilon)$ onto the separable metric space $S$. For each $x \in X$, let $U(x)=G(x) \cap B(x)$, where $G(x) \in \mathscr{G}_{\omega_{1}}(x)$ and $B(x) \in \mathscr{B}_{\mathbf{R}}$ such that $x \in B(x)$. Now, for each $x \in X$, let $V(x)$ be an open set in $(X, \Upsilon)$ such that $x \in V(x)$ and if $y \in \overline{f(V(x))}$ in $S$, then $f^{-1}(y) \in U(x)$. Furthermore, without loss of generality, for each $x \in X$, assume $V(x)=G^{\prime}(x) \cap B^{\prime}(x)$ where $G^{\prime}(x) \in \mathscr{G}_{\omega_{1}}(x)$ and $x \in B^{\prime}(x) \in \mathscr{B}_{\mathbf{R}}$. By the Pressing Down Lemma, there exist $\gamma<\omega_{1}$ and a stationary subset $Y$ of $X$ in $(X, \Upsilon)$ such that for all $x=x_{\alpha} \in Y, G^{\prime}(x)=\left\{x_{\beta} \mid \gamma<\beta \leqslant \alpha\right\}$. Furthermore, there exist $Y^{\prime} \subseteq Y$ and $B \in \mathscr{B}_{\mathbf{R}}$ such that $Y^{\prime}$ is stationary in $(X, \Upsilon)$ and for each $x \in Y^{\prime}, B^{\prime}(x)=B$. Consider $f\left(Y^{\prime}\right)$ in $S$. Since $S$ is separable, there exists a countable subset $J$ of $f\left(Y^{\prime}\right)$ such that $f\left(Y^{\prime}\right)=\bar{J}$ in $S$. However, by construction, there exists $x \in Y^{\prime}$ such that $f^{-1}(J) \subseteq V(x)$. Hence, $\overline{V(x)}$ in $S$ is uncountable. Since $U(x)$ is countable, we have a contradiction to the assumption that $f$ is regular.

THEOREM 1. If $(X, \Upsilon) \in \mathscr{C}$, then $(X, \Upsilon)$ is a completely regular, submetrizable collectionwise Hausdorff, pseudo-normal, countably metacompact space with a base of countable order and a $G_{\delta^{-}}$diagonal. However, $(X, \Upsilon)$ is neither $\theta$-refinable, subparacompact, metalindelöf, cometrizable, nor locally compact.

THEOREM 2. (MA $+\neg \mathrm{CH})$ If $(X, \Upsilon) \in \mathscr{C}$, then $(X, \Upsilon)$ is perfect. 
Proof. A space is perfect provided each of its closed subsets is a $G_{\delta}$-set. Under (MA) each separable metrizable space of cardinality $<c$ has the property that each of its subsets is a $G_{\delta}$-set $[\mathbf{S}, \mathbf{T}]$. Hence, it follows immediately that each subset of $(X, \Upsilon)$ is a $G_{\delta}$-set in $(X, \Upsilon)$.

\section{Normality in the class $\mathscr{C}$.}

Lemma 13. If $(X, \Upsilon) \in \mathscr{C}$ and $\mathscr{H}$ is a discrete collection of closed sets in $(X, \Upsilon)$, then at most countably many members of $\mathscr{H}$ are not $\sigma$-discrete.

Proof. Suppose $\mathscr{H}$ is an uncountable, discrete collection of closed sets in $(X, \Upsilon)$ such that each member of $\mathscr{H}$ is not $\sigma$-discrete (and hence, by Lemma 3 , stationary in $\left.\left(X, \Upsilon_{\omega_{1}}\right)\right)$. For each $H \in \mathscr{H}$ and $x \in H$, let $U(x)=G(x) \cap B(x)$ such that $G(x) \in \mathscr{G}_{\omega_{1}}(x), \quad x \in B(x) \in \mathscr{B}_{\mathbf{R}}$, and $U(x) \cap(\bigcup\{K \in \mathscr{H} \mid K \neq H\})=\varnothing$. Now, for each $H \in \mathscr{H}, \exists Y_{H} \subseteq H$ and $B(H) \in \mathscr{B}_{\mathbf{R}}$ such that $Y_{H}$ is stationary in $(X, \Upsilon)$ and $B(x)=B(H)$ for each $x \in Y_{H}$. Let $U(H)=\bigcup\left\{U(x) \mid x \in Y_{H}\right\}$. Now, $\exists B \in \mathscr{B}_{\mathbf{R}}$ such that $B(H)=B$ for uncountably many $H$. However, by Lemma 4,

$$
|B(H) \backslash U(H)|<\omega_{1}
$$

for each such $H$. This is a contradiction since if $B(H)=B(K)$, then $\left|U(H) \cap Y_{K}\right|$ $>\omega$ where $Y_{K} \subseteq K$.

LEMMA 14. If $(X, \Upsilon) \in \mathscr{C}$ and $H$ and $K$ are disjoint closed sets in $(X, \Upsilon)$, then $H=\bigcup\left\{H_{i}^{1} \mid i<\omega\right\} \cup \cup\left\{H_{i}^{2} \mid i<\omega\right\}$, where for each $i<\omega, K$ and $H_{i}^{1}$ can be separated by open sets in $(X, \Upsilon)$ and $H_{i}^{2}$ is closed and discrete in $(X, \Upsilon)$.

Proof. Suppose $H$ and $K$ are disjoint closed sets in $(X, \Upsilon)$. If $H$ is not stationary in $\left(X, \Upsilon_{\omega_{1}}\right)$, we are finished since $H$ is $\sigma$-discrete in $(X, \Upsilon)$ and metrizable by Lemma 3. Hence, suppose $H$ is stationary. For each $x \in H$, let $U(x)=G(x) \cap B(x)$ such that $G(x) \in \mathscr{G}_{\omega_{1}}(x), x \in B(x) \in \mathscr{B}_{\mathbf{R}}$, and $\overline{U(x)} \cap K=\varnothing$ in $(X, \Upsilon)$. Let $U(H)=$ $\bigcup\{U(x) \mid x \in H\}$, and note that $U(H) \cap K=\varnothing$. Now, let $W=\{B \in \mathscr{B} \mid B=B(x)$ for each $x$ in some stationary subset $Y_{H}$ of $H$ w.r.t. $\left.\left(X, \Upsilon_{\omega_{1}}\right)\right\}$. It follows that $H \backslash(\bigcup W \cap H)$ is closed and $\sigma$-discrete in $(X, \Upsilon)$ by Lemma 4 . Furthermore, since $H \backslash(\cup W \cap H)$ is contained in an open metrizable subset of $(X, \Upsilon)$ by Lemma 3 , it follows that $H \backslash(\bigcup W \cap H)=\bigcup\left\{H_{i}^{2} \mid i<\omega\right\}$, where for each $i, H_{i}^{2}$ is closed and discrete in $(X, \Upsilon)$. Now, by Lemma 4 , it follows that for each $B \in W,|B \cap K|<\omega_{1}$. It also follows that $|\bar{B} \cap K|<\omega_{1}$. For suppose not, then $\exists J \subseteq(\bar{B} \cap K) \backslash B$ such that $|J|=\omega_{1}$. However, $J$ has a limit point $x$ in $Y_{H}$ w.r.t. $\left(X, \Upsilon_{\omega_{1}}\right)$. Let $y \in G(x) \cap$ $J$, then $y \in \overline{U(x)} \cap K$, which contradicts the choice of $U(x)$. Hence, enumerate $W=\left\{W_{i} \mid i<\omega\right\}$. Then for each $i<\omega_{1}$, let $Q_{i}=\bar{W}_{i}$ in $(X, \Upsilon)$ and let $H_{i}^{1}=Q_{i} \cap H$. Since for each $i<\omega, Q_{i} \cap K$ is countable and $(X, \Upsilon)$ is pseudo-normal, $\exists \sigma$-disjoint open sets $V_{i}$ and $V_{k}$ containing $H_{i}^{1}$ and $Q_{i} \cap K$, respectively. It follows that $H_{i}^{1} \subseteq W_{i} \cap V_{i}$ and $\left(\overline{W_{i} \cap V_{i}}\right) \cap K=\varnothing$ in $(X, \Upsilon)$. Thus, $H=\bigcup\left\{H_{i}^{1} \mid i<\omega\right\} \cup$ $\cup\left\{H_{i}^{2} \mid i<\omega\right\}$ as required.

LEMMA $15[\mathbf{Z}]$. The $T_{1}$-space $X$ is normal provided that if $H$ and $K$ are disjoint closed sets in $X$, then $\exists\left\{U_{i} \mid i<\omega\right\}$ such that for each $i<\omega, U_{i}$ is an open set in $X$ containing $H$, and $\cap\left\{\bar{U}_{i} \cap K \mid i<\omega\right\}=\varnothing$. 
THEOREM 3. If $(X, \Upsilon) \in \mathscr{C}, T F A E$

(1) $X$ is strongly collectionwise Hausdorff.

(2) $X$ is countably paracompact.

(3) $X$ is normal.

(4) $X$ is collectionwise normal.

PROof. (3) $\leftrightarrow$ (4). Each collectionwise normal space is normal. Hence, suppose $(X, \Upsilon)$ is normal. Let $\mathscr{H}$ denote a discrete collection of closed sets in $(X, \Upsilon)$. By Lemma 13, at most countably many members of $\mathscr{H}$ are not $\sigma$-discrete. Let $\mathscr{H}_{1}=\{H \in \mathscr{H} \mid H$ is not $\sigma$-discrete $\}$, and let $\mathscr{H}_{2}=\{H \in \mathscr{H} \mid H$ is $\sigma$-discrete $\}$. Since $(X, \Upsilon)$ is normal, $\exists$ disjoint open sets $U$ and $V$ in $(X, \Upsilon)$ containing $\cup \mathscr{H}_{1}$ and $\cup \mathscr{H}_{2}$ respectively. Since $\mathscr{H}_{1}$ is countable and $(X, \Upsilon)$ is normal, we can clearly screen $\mathscr{H}_{1}$ with open sets in $(X, \Upsilon)$ contained in $V$. Now, by Lemma 3 , there exists an open metrizable subset $W$ of $(X, \Upsilon)$ containing $\cup \mathscr{H}_{2}$ such that $W \subseteq V$. Hence $\mathscr{H}_{2}$ can be screened within $W$, and $\mathscr{H}=\mathscr{H}_{1} \cup \mathscr{H}_{2}$ can be screened by open sets in $(X, \Upsilon)$.

(3) $\rightarrow(2)$. $(X, \Upsilon)$ is countably metacompact by Theorem 1 . Each normal, countably metacompact $T_{2}$-space is countably paracompact [K, D].

(2) $\rightarrow$ (1). A space $X$ is strongly collectionwise Hausdorff provided each closed discrete subset of $X$ can be screened by a discrete collection of open sets in $X$. Hence, suppose $(X, \Upsilon)$ is countably paracompact and let $K$ denote a closed, discrete subset of $(X, \Upsilon)$. Since $(X, \Upsilon)$ is collectionwise Hausdorff, let $\mathscr{U}$ denote a collection of pairwise disjoint open sets in $(X, \Upsilon)$ screening $K$. Consider $K$ and $H=X \backslash \bar{\cup}$. By Lemma 14, $H=\bigcup\left\{H_{i}^{1} \mid i<\omega\right\} \cup \cup\left\{H_{i}^{2} \mid i<\omega\right\}$, where for each $i<\omega, K$ and $H_{i}^{1}$ can be separated by open sets in $(X, \Upsilon)$ and $H_{i}^{2}$ is closed and discrete in $(X, \Upsilon)$. However, since $K$ is discrete in $(X, \Upsilon)$ and $(X, \Upsilon)$ is collectionwise Hausdorff, $K$ and $H_{i}^{2}$ can also be separated by open sets in $(X, \Upsilon)$ for each $i<\omega$. Thus, $H$ is a closed subset of $(X, \Upsilon)$ covered by a countable collection $\mathscr{V}$ of open sets in $(X, \Upsilon)$ such that the closure of each member of $\mathscr{V}$ in $(X, \Upsilon)$ misses $K$. Since $(X, \Upsilon)$ is countably paracompact, $\exists$ a locally finite refinement $\mathscr{V}^{\prime}$ of $\mathscr{V}$ covering $H$. It follows that $\left\{U \backslash\left(\overline{U_{\mathscr{V}^{\prime}}} \cap U\right) \mid U \in \mathscr{U}\right\}$ is a discrete collection of open sets in $(X, \Upsilon)$ screening $K$.

$(1) \rightarrow(4)$. Suppose $(X, \Upsilon)$ is strongly collectionwise Hausdorff, and $H$ and $K$ are disjoint closed sets in $(X, \Upsilon)$. Then, by Lemma 14, there exist $\left\{U_{i} \mid i<\omega\right\}$ satisfying Lemma 15. Hence, $(X, \Upsilon)$ is normal.

Example 1. $\exists(X, \Upsilon) \in \mathscr{C}$ such that $(X, \Upsilon)$ is not normal.

Proof. Let $C$ be a Cantor set in $\mathbf{R}, M \subseteq C$, and $|M|=\omega_{1}$. Let $Y \subseteq \mathbf{R} \backslash C$ such that $|Y|=\omega_{1}$ and $Y$ is uncountable in each open interval in $\mathbf{R}$. Let $\mathscr{B}$ denote a countable basis for $\left(X, \Upsilon_{\mathbf{R}}\right)$, and for each $x \in X$, let $\left\{B_{i}(x) \mid i<\omega\right\}$ denote a nonincreasing local base at $x$ from $\mathscr{B}$. For each $x \in M$ and $i<\omega$, let $S_{i}(x)$ denote a countably dense subset of $B_{i}(x)$ such that (1) $S_{i}(x) \subseteq Y$, (2) if $i \neq j$, then $S_{i}(x) \cap S_{j}(y)=\varnothing$ for all $x, y \in M$, and (3) if $x \neq y$, then $S_{i}(x) \cap S_{j}(y)=\varnothing$ for all $i, j<\omega$. Let $H=M \cup \cup\left\{S_{i}(x) \mid i<\omega\right.$ and $\left.x \in M\right\}$.

Now, we define an $\omega_{1}$-type order on $X$ as follows. Let $L_{1}=$ the set of all ordinals in $\omega_{1}$ which are limits of nonlimit ordinals but not limits of limit ordinals. Let $L_{2}=$ the set of all ordinals in $\omega_{1}$ which are limits of $L_{1}$ but which are not limits of 
limits of $L_{1}$. Note that $L_{2}$ is discrete, hence, by (2) $\rightarrow(6)$ of Lemma $3, L_{2}$ is contained in an open metrizable subset of $\omega_{1}$ with the order topology. Hence, for each $\alpha \in L_{2}$, let $G(\alpha)$ denote a basic open set in $\omega_{1}$ containing $\alpha$ such that if $\alpha \neq \beta$, then $G(\alpha) \cap G(\beta)=\varnothing$. Furthermore for each $\alpha \in L_{2}$, let $\left\{\alpha_{i} \mid i<\omega\right\} \subseteq G(\alpha)$ be such that $\left\{\alpha_{i}\right\}_{i<\omega}$ converges to $\alpha$ in $\omega_{1}$, and for each $i<\omega, \alpha_{i} \in L_{1}$. Now for each $\alpha \in L_{2}$ and $0<i<\omega$, let $G_{i}(\alpha)=\left\{\beta \in G(\alpha) \mid \alpha_{i-1}<\beta \leqslant \alpha_{i}\right.$ in $\left.\omega_{1}\right\}$, and let $G_{0}(\alpha)=\left\{\beta \in G(\alpha) \mid \beta \leqslant \alpha_{0}\right\}$. We define an $\omega_{1}$-type order on $X$ as follows. Identify the points of $M$ and the ordinals of $L_{2}$ in a one-to-one fashion, i.e., $M=\left\{x_{\alpha} \mid \alpha\right.$ $\left.\in L_{2}\right\}$. Then for each $x_{\alpha} \in M$ and $i<\omega$, identify the points of $S_{i}\left(x_{\alpha}\right)$ and those of $G_{i}(\alpha)$ in a one-to-one fashion. Finally, identify the points of $X \backslash H$ and $\left\{\omega_{1} \backslash \bigcup\left\{G(\alpha) \mid \alpha \in L_{2}\right\}\right.$ in a one-to-one fashion such that for each $B \in \mathscr{B}$, some subset of $B$ is identified with a stationary set in $\omega_{1}$. Let $\left(X, \Upsilon_{\omega_{1}}\right)$ denote $X$ with the topology induced by this $\omega_{1}$-type ordering.

Consider $(X, \Upsilon)$, the intersection topology w.r.t. $\left(X, \Upsilon_{\mathbf{R}}\right)$ and $\left(X, \Upsilon_{\omega_{1}}\right)$. Clearly, $M$ and $X \backslash H$ are disjoint closed sets in $(X, \Upsilon)$. However, $M$ and $X \backslash H$ cannot be separated by open sets in $(X, \Upsilon)$. To see that this is true, suppose there exist open sets $V$ and $W$ in $(X, \Upsilon)$ such that $M \subseteq V$ and $(X \backslash H) \subseteq W$ with $V \cap W=\varnothing$. For each $x=x_{\alpha} \in M$ and $i<\omega$, let $U_{i}(x)=\left\{x_{\beta} \mid \beta \in G_{i}(\alpha)\right\}$. Now, for each $x \in M$, $\exists i_{x}<\omega$ such that $x \in\left(U_{i_{x}}(x) \cap B_{i_{x}}(x)\right) \subseteq V$. Hence, for some $i<\omega$ and $B \in \mathscr{B}$, $B=B_{i_{x}}$ and $i=i_{x}$ for uncountably many $x \in M$. Denote by $M^{\prime}$ the set of all such $x$. Now, $\exists$ a stationary subset of $X \backslash H$ w.r.t. $\left(X, \Upsilon_{\omega_{1}}\right)$ contained in $B$. Thus, $\exists q \in(X \backslash H) \cap B$ such that $q$ is a limit point of $M^{\prime}$ in $\left(X, \Upsilon_{\omega_{1}}\right)$. But, if $G(q)$ is an open set in $\left(X, \Upsilon_{\omega_{1}}\right)$ containing $q$, then $S_{i}(x) \subseteq G(q)$ for some $x \in M^{\prime}$ where $S_{i}(x)$ is dense in $B$ w.r.t. $\left(X, \Upsilon_{\mathbf{R}}\right)$. Since $q \in B$, it follows that $q$ must be a limit point of $V$ in $(X, \Upsilon)$. This contradicts the assumption that $M$ and $X \backslash H$ can be separated in $(X, \Upsilon)$. Hence $(X, \Upsilon)$ is not normal.

ExAmple 2. (CH) $\exists(X, \Upsilon) \in \mathscr{C}$ such that $(X, \Upsilon)$ is $\omega_{1}$-compact and collectionwise normal, but not perfect.

Proof. Under $\mathrm{CH}, \exists$ a Lusin set $X \subseteq \mathbf{R}[\mathbf{L}, \mathbf{M}]$. A Lusin set is an uncountable subset $X$ of $\mathbf{R}$ such that each uncountable subset of $X$ is dense in some interval. Note that, in fact, for each uncountable subset $Y$ of $X$ there exists an open interval in $\mathbf{R}$ such that $Y$ is uncountable in each subinterval. To see that this is true, consider $Y^{\prime}=\{x \in Y \mid$ each open set in $\mathbf{R}$ containing $x$ contains uncountably many points of $Y$ \}. Clearly, $Y^{\prime}$ is uncountable. Hence $\exists$ an open interval in $\mathbf{R}$ in which $Y^{\prime}$ is dense. Since each subinterval contains a point of $Y^{\prime}$, it must also contain uncountably many points of $Y$.

Let $\mathscr{B}$ denote a countable basis for $\left(X, \Upsilon_{\mathbf{R}}\right)$, and let $\left(X, \Upsilon_{\omega_{1}}\right)$ be such that $X$ is stationary in each element of $\mathscr{B}$ w.r.t. $\left(X, \Upsilon_{\omega_{1}}\right)$. It follows that $(X, \Upsilon)$ is $\omega_{1}$-compact, i.e. each closed discrete subset of $(X, \Upsilon)$ is countable. To see that this is true, suppose not and let $K$ denote an uncountable, closed, and discrete subset of $(X, \Upsilon)$. Then there exists $B_{K} \in \mathscr{B}$ such that $K$ is uncountable in each element of $\mathscr{B}$ contained in $B_{K}$. However, $\exists Y \subseteq B_{K}$ such that $Y$ is stationary w.r.t. $\left(X, \Upsilon_{\omega_{1}}\right)$ and $Y \cap K=\varnothing$. Hence, for each $x \in Y, \exists G(x)$ in $\left(X, \Upsilon_{\omega_{1}}\right)$ and $B(x) \in \mathscr{B}$ such that $B(x) \subseteq B_{K}$, $x \in G(x) \cap B(x)$, and $(G(x) \cap B(x)) \cap K=\varnothing$. Thus, for some $B \in \mathscr{B}, B=B(x)$ for each $x$ in a subset $Y^{\prime}$ of $Y$ such that $Y^{\prime}$ is also stationary w.r.t. $\left(X, \Upsilon_{\omega_{1}}\right)$. Now, 
since $K$ is uncountable in $B, \exists q \in Y^{\prime}$ such that $q$ is a limit point of $B \cap K$ w.r.t. $\left(X, \Upsilon_{\omega_{1}}\right)$. Thus, $(G(q) \cap B) \cap K \neq \varnothing$ which is a contradiction.

Since each closed discrete subset of $(X, \Upsilon)$ is countable, and $(X, \Upsilon)$ is pseudo-normal $(X, \Upsilon)$ is strongly collectionwise Hausdorff. Hence, by Theorem 2, $(X, \Upsilon)$ is collectionwise normal.

$(X, \Upsilon)$ is not perfect, since in a perfect space each discrete set is the union of countably many closed and discrete subsets. However, there is an uncountable subset $K$ of $X$ such that $K$ is identified with the nonlimit ordinals in $\omega_{1}$ and hence $K$ is discrete in $(X, \Upsilon)$. If $K$ were the union of countably many closed and discrete sets, $K$ would be countable since $X$ is $\omega_{1}$-compact.

V. Concluding remarks. In this paper, the author has presented a study of the intersection topology w.r.t. a given subset of the real line and a given order topology of type $\omega_{1}$ on that set. Surprisingly, it was shown that the normality of the intersection topology apparently depends on both the topological characteristics of the subset and the particular choice of an $\omega_{1}$-type order. The author gave several applications which suggest that such spaces are a useful tool in the analysis of topological structure.

As noted in §I, all the results in the paper are true for subsets of an arbitrary separable metric space. The author views these results as a foundation for a systematic study of the intersection topology. He hopes to present further results and applications at a later date.

Some generalities. If $P$ is either $T_{1}, T_{2}, T_{3}, T_{3-1 / 2}$, first countable, second countable, or metrizable, then the intersection of two topologies on the set $X$ each having property $\mathbf{P}$ also has property $\mathrm{P}$. Under $\mathrm{CH}$, the intersection of the Michael line topology on $\mathbf{R}$ and an order topology of type $\omega_{1}$ on $\mathbf{R}$ is metrizable. Hence, the intersection of a nonmetrizable $T_{3}$ topology and an $\omega_{1}$-type order topology on a given set can produce a metrizable topology.

ACKNOWLEDGMENTS. Obviously, this paper was inspired by the elegant constructions in [Po and PP]. In addition, the author's initial interest in the intersection topology owes much to his interaction with Eric van Douwen in working through the examples and techniques in [Po]. The author wishes to acknowledge support under a Research Fellowship from the Futuretrends Foundation and an ORS grant from the Committee of Vice-Chancellors and Principles of the Universities of the United Kingdom. Certain of the work contained herein was accomplished while the author was a Fellow of the Institute for Medicine and Mathematics at Ohio University and a Visiting Fellow at St. Edmund Hall, Oxford.

ADDED IN REVISION. The author's questions concerning the existence of a perfectly normal member of the class $\mathscr{C}$ have recently been answered by Ken Kunen. Kunen has elegantly shown that no member of $\mathscr{C}$ can be both perfect and normal. Furthermore, he has shown that there is a model of set theory in which some members of $\mathscr{C}$ are perfect and others are normal. These results are to appear in [K. Kunen, On ordinal metric intersection topologies].

ADDED IN PROOF. The author has established that for each $(X, \Upsilon) \in \mathscr{C}$, the square of $X(X, \Upsilon)$ is not normal. Hence, under MA $+\neg \mathrm{CH}$, we have a new class of normal Moore spaces whose squares are not normal. 


\section{REFERENCES}

[AP] K. Alster and R. Pol, Moore spaces and collectionwise Hausdorff property, Bull. Acad. Polon. Sci. Ser. Math. Astronom. 23 (1975), 1189-1192.

[B] R. H. Bing, Metrization of topological spaces, Canad. J. Math. 3 (1951), 175-186.

[Ch] J. Chaber, Conditions which imply compactness in countably compact spaces, Bull. Acad. Polon. Sci. Ser. Math. Astronom. 24 (1976), 993-998.

[Co] H. Cook, Cartesian products and continuous semi-metrics, preprint.

[D] C. H. Dowker, On countably paracompact spaces, Canad. J. Math. 3 (1961), 219-224.

[vDL] E. K. van Douwen and D. J. Lutzer, On the classification of stationary sets, Michigan Math. J. 26 (1979), 47-64.

[F] G. Fodor, Fine Bemerkung zur Theorie der regressiven Funktionen, Acta. Sci Math. (Szeged) 17 (1956), 139-142.

[HJ] A. Hajnal and I. Juhász, On hereditarily $\alpha$-Lindelöf and hereditarily $\alpha$-separable spaces, Ann. Univ. Sci. Budapest 9 (1968), 115-124.

[K] M. Katětov, Measures in fully normal spaces, Fund. Math. 38 (1951), 73-84.

[L] N. Lusin, Sur un probleme de M. Baire, C. R. Hebdomadaires Seances Acad. Sci. Paris 158 (1914), 1258-1261.

[M] P. Mahlo, Über Teilmengen des Kontinuums von dessen Machtigkeit, Sitzungsberichte der Sachsischen Akademie der Wissenschaften zu Leipzig, Mathematish-Naturwissenschaftliche Klasse 65, pp. 283-315.

[Po] R. Pol, A perfectly normal locally metrizable non-paracompact space, Fund. Math. 97 (1977), 37-42.

[PP] E. Pol and R. Pol, $A$ hereditarily normal strongly zero-dimensional space with a subspace of positive dimension and an N-compact space of positive dimension, Fund. Math. 97 (1977), 43-50.

[Pr] T. Przymusinski, Normality and separability of Moore spaces, Set-Theoretic Topology, Academic Press, New York, 1977, pp. 325-337.

[R $\left.\mathbf{R}_{1}\right]$ G. M. Reed, Q-sets and Shelah's Principle, Notices Amer. Math. Soc. 26 (1979), A124.

$\left[\mathbf{R}_{2}\right]$ _. A note on duality in $S$ and $L$ spaces, Abstracts Amer. Math. Soc. 2 (1981), 178-179.

$\left[\mathbf{R}_{3}\right]$, Submetrizable topologies on the ordinals, Topology and Order Structures (H. R. Bennett and D. J. Lutzer, eds.), Math. Centre Tract 169, Math. Centrum, Amsterdam, 1984, pp. 63-64.

$\left[\mathbf{R}_{4}\right] \ldots$, On normality and countable paracompactness, Notices Amer. Math. Soc. 22 (1975), A126.

$\left[\mathbf{R}_{5}\right]$, Collectionwise Hausdorff versus collectionwise normal with respect to compact sets, Topology Appl. 16 (1983), 259-272.

[R6 _. On continuous images of Moore spaces, Canad. J. Math. 26 (1974), 1475-1479.

$\left[\mathbf{R}_{7}\right]$. On chain conditions in Moore spaces, General Topology Appl. 4 (1974), 255-267.

$\left[\mathbf{R}_{\mathbf{8}}\right]$ - Concerning first countable spaces, III, Trans. Amer. Math. Soc. 210 (1975), 169-177.

$\left[\mathbf{R}_{\mathbf{9}}\right]$ On the existence of point-countable bases in Moore spaces, Proc. Amer. Math. Soc. 45 (1974), 437-440.

$\left[\mathbf{R}_{10}\right] \ldots$, On subspaces of separable first countable $T_{2}$-spaces, Fund. Math. 91 (1976), 199-211.

[S] T. Shinoda, Some consequences of Martin's Axiom and the negation of the continuum hypothesis, Nagoya Math. J. 49 (1973), 117-125.

[T] F. D. Tall, Set-theoretic consistency results and topological theorems concerning the normal Moore space conjecture and related problems, $\mathrm{Ph}$. D. Thesis, Univ. of Wisconsin, 1969.

[U] S. Ulam, Zur masstheorie in der allgeneinen Mengenlehre, Fund. Math. 16 (1930), 141-150.

[Wa] M. L. Wage, A collectionwise Hausdorff nonnormal Moore space, Canad. J. Math. 28 (1976), 632-634.

[Wo] J. M. Worrell, Isolated sets of points in non-metrizable spaces, Notices Amer. Math. Soc. 11 (1964), 250.

[WW] J. M. Worrell and H. H. Wicke, Characterizations of developable topological spaces, Canad. J. Math. 17 (1965), 820-830.

[Z] P. Zenor, On countable paracompactness and normality, Prace Math. 13 (1969), 23-32.

Department of Mathematics, Ohio University, Athens, OHio 45701

Current address: Oxford University Computing Laboratory, Programming Research Group, 8-11 Keble Road, Oxford OX1 3QD, England 